\title{
Gait analysis before and after corrective osteotomy in patients with knee osteoarthritis and a valgus deformity
}

\author{
N. van Egmond ${ }^{1} \cdot$ N. Stolwijk ${ }^{2} \cdot$ R. van Heerwaarden ${ }^{3}$ A. $\operatorname{van}_{\text {Kampen }^{1}}$. \\ N. L. W. Keijsers ${ }^{4}$
}

Received: 4 May 2015 / Accepted: 4 February 2016 / Published online: 18 February 2016

(c) The Author(s) 2016. This article is published with open access at Springerlink.com

\begin{abstract}
Purpose In this prospective study, the changes in kinetics and kinematics of gait and clinical outcomes after a varus osteotomy (tibial, femoral or double osteotomy) in patients with osteoarthritis (OA) of the knee and a valgus leg alignment were analysed and compared to healthy subjects.

Methods Twelve patients and ten healthy controls were included. Both kinetics and kinematics of gait and clinical and radiographic outcomes were evaluated.

Results The knee adduction moment increased significantly postoperatively $(p<0.05)$ and almost similar to the control group. Patients showed less knee and hip flexion/ extension motion and moment during gait pre- and postoperatively compared to the controls. A significant improvement was found in WOMAC [80.8 (SD 16.1), $p=0.000]$, KOS [74.9 (SD 14.7), $p=0.018]$, OKS [21.2 (SD 7.5), $p=0.000]$ and VAS-pain [32.9 (SD 20.9), $p=0.003$ ] in all patients irrespective of the osteotomy technique used. The radiographic measurements showed a mean hip knee ankle (HKA) angle correction of $10.4^{\circ}$ (95\% CI 6.4 $\left.-14.4^{\circ}\right)$.

Conclusion In patients with knee OA combined with a valgus leg alignment, the varus-producing osteotomy is a
\end{abstract}

N. van Egmond

nienkevanegmond@hotmail.com

1 Department of Orthopaedics, Radboud University Medical Centre, P.O. Box 9101, 6500, HB, Nijmegen, The Netherlands

2 Institute of Health Studies, HAN University, Kapittelweg 33, 6525, EN, Nijmegen, The Netherlands

3 Department of Orthopaedics, Sint Maartenskliniek, P.O. Box 9011, 6500, GM, Nijmegen, The Netherlands

4 Research Department, Sint Maartenskliniek, P.O. Box 9011, 6500, GM, Nijmegen, The Netherlands successful treatment. Postoperatively, the patients showed kinetics and kinematics of gait similar as that of a healthy control group. A significant increase in the knee adduction moment during stance phase was found, which was related to the degree of correction. The HKA angle towards zero degrees caused a medial shift in the dynamic knee loading. The medial shift will optimally restore cartilage loading forces and knee ligament balance and reduces progression of OA or the risk of OA. A significant improvement in all clinical outcomes was also found.

Level of evidence III.

Keywords Double osteotomy $\cdot$ Supracondylar femoral osteotomy · Closed wedge medial high tibial osteotomy · Valgus alignment $\cdot$ Gait analysis

\section{Introduction}

Malalignment of the leg increases the risk of progression of knee osteoarthritis (OA) and causes a decline in physical function and progression of pain $[15,32]$. One of the possible reasons for this increased risk of OA is that a malalignment of the knee influences the forces and moments acting on the knee during walking. In patients with medial knee OA and a varus alignment, an increased knee adduction moment is typically observed [17, 35, 36]. Kaufman et al. [17] found a significant difference between patients with knee OA (0.39\% BW-HT, SD 0.28) and healthy subjects $(0.36 \% \mathrm{BW}-\mathrm{HT}$, SD 0.36). Turcot et al. [35] found a significant difference between patients with a varus leg alignment $(0.62 \mathrm{Nm} / \mathrm{kg}, \mathrm{SD} 0.19)$ compared to the control group $(0.50 \mathrm{Nm} / \mathrm{kg}$, SD 0.12). Moreover, the literature has shown a relationship between the degree of knee deformity and the forces acting on the knee [32, 33, 35, 36, 39, 40]. 
Weidenhielm et al. [39] found correlations between the hip knee ankle (HKA) angle and the peak adduction moment before surgery, after surgery and between the change in HKA angle and the change in peak adduction moment after surgery. Furthermore, varus alignment and increased knee adduction moment were associated with the progression of OA [25, 26, 32]. Sharma et al. [32] found a significant correlation between adduction moment and the Kellgren-Lawrence grade in knees. They also found significant correlations between adduction moment and joint space width in knees. In another study, Sharma and Song [33] found that a varus alignment was associated with a fourfold increase in the odds of medial progression (adjusted odds ratio 4.09, $95 \%$ CI 2.20-7.62). Hence, malalignment of the leg alters the kinetics and kinematics in the knee, which most likely increases the risk of knee OA [32, 35].

When conservative treatment is no longer successful, corrective osteotomy is considered for young and active
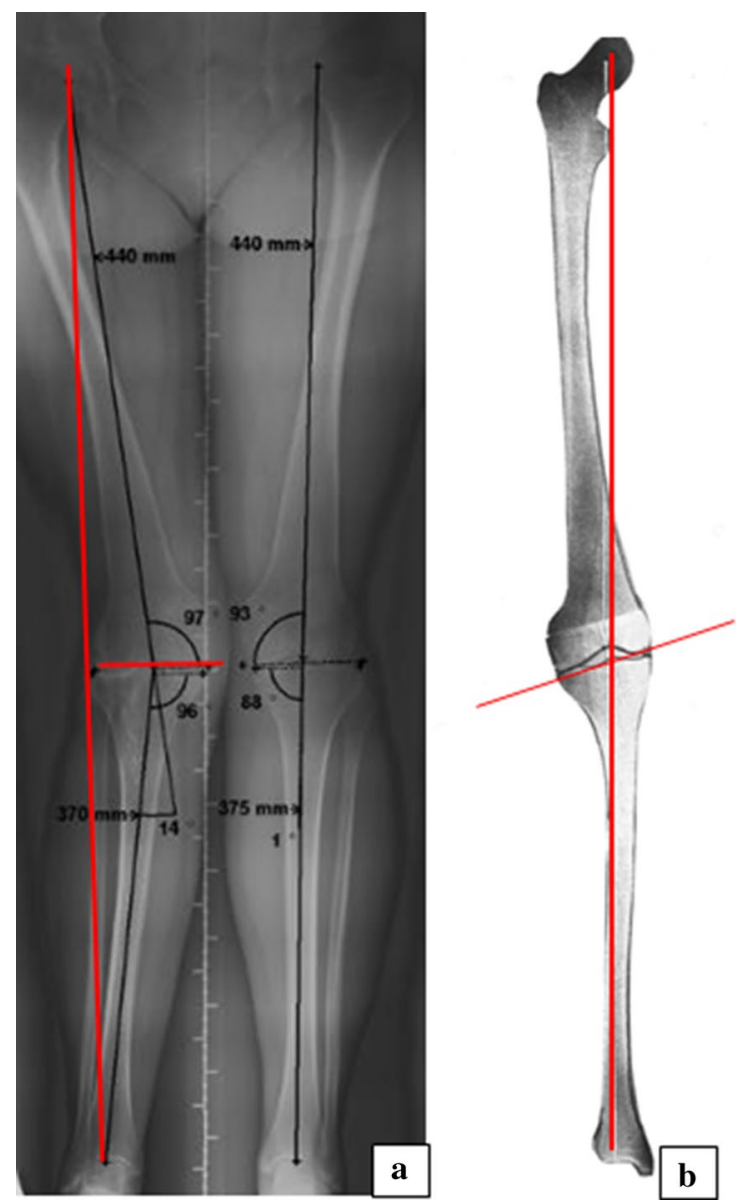

Fig. 1 Rationale of double osteotomy in valgus corrective surgery. Weight-bearing long-leg radiographs and planning drawings including weight-bearing lines (WBL) and knee joint orientation lines (KJOL) of one of the study patients. a Preoperative valgus leg alignment caused by femoral and tibial bone deformity, WBL lateral and KJOL neutral. b Planning drawing of medial closing wedge distal patients with lateral knee $\mathrm{OA}$ and a valgus leg alignment [15]. The purpose of a correction osteotomy is to realign the weight-bearing lines while maintaining normal knee joint line orientation (Fig. 1) [1, 2, 6, 7, 9-11, 14, 22, 23, $27,28,30,34,37]$. A corrective osteotomy can be performed in either the femur or tibia or in both bones, i.e. a double osteotomy.

The kinetic and kinematics of gait of a varus medial osteoarthritic knee and the effect of a valgus osteotomy on these gait characteristics are well described in the literature $[8,17,21,25,35,36-39]$. It is proven that a valgus-producing osteotomy is able to improve the kinetics and kinematics of gait [21,38], causing improvements in clinical results and quality of life [4, 14]. Lind et al. [21] found a significant increase in walking speed, maximum knee flexion and a significant decrease in the mean maximum adduction moment after a valgus-producing osteotomy. Some literature addressed that the amount of adduction
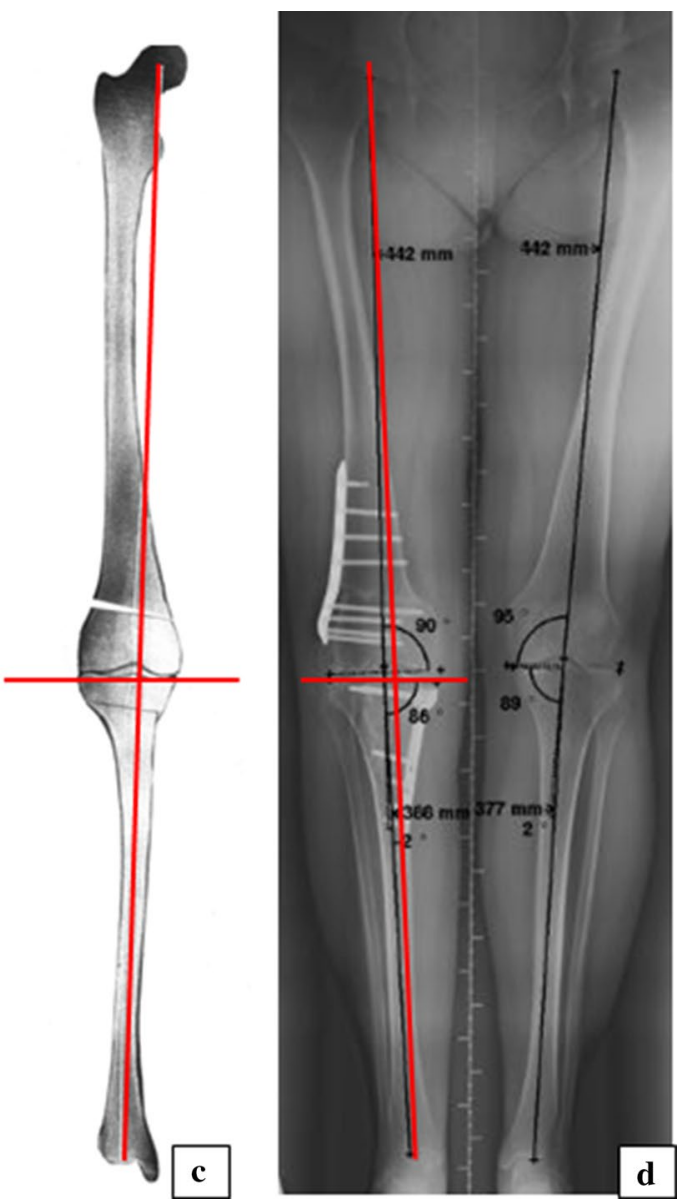

femur osteotomy resulting in neutral WBL and severe valgus KJOL. c Planning drawing of double osteotomy, i.e. lateral open wedge distal femur and medial closing proximal tibial osteotomy, resulting in neutral WBL and neutral KJOL. d Postoperative leg alignment after double osteotomy 
moment is a predictive value for the clinical results after a valgus osteotomy. Patients with a higher adduction moment showed inferior clinical results compared to patients with a lower adduction moment [29, 38]. Also, the improvements in kinetics and kinematics of gait following a valgus osteotomy decrease the rate of the progression of medial knee $\mathrm{OA}$, thereby delaying or preventing later conversion to a knee arthroplasty.

Although the kinetics and kinematics of gait in a medial varus osteoarthritic knee and the effect of a valgus osteotomy are well described $[8,36,38,39]$, the effect of a varus osteotomy on gait has been investigated only once [6]. In that study, only one parameter, the knee peak adduction moment, was studied in a subgroup of 12 patients with a lateral open wedge high tibial osteotomy and a mild valgus malalignment [mean HKA angle $2.4^{\circ}$ (SD 2.4)] without an abnormal mechanical lateral distal femoral angle (mLDFA). The authors found a significant increase in the peak knee adduction moment during gait (mean change $(95 \% \mathrm{CI})$ of $0.72 \% \mathrm{BW} * \mathrm{Ht}(0.42,1.02)$ suggesting a medial shift in dynamic knee joint load. Although the peak adduction moment is an important outcome, it is a simplification of describing the effect of a varus osteotomy on gait. Detailed kinetics and kinematics of gait after a varus osteotomy have not yet been described in the literature. The spatiotemporal parameters, the flexion/extension angles, the abduction/adduction angles, the flexion/ extension moments, the abduction/adduction moments of the knee and hip during the whole stance phase are important parameters in gait studies [24]. Furthermore, in contrast to the study of Collins et al. [6], patients with a large HKA angle and with an abnormal mLDFA were included. As a consequence, patients who underwent a medial closing wedge high tibial osteotomy (TKO), a medial closing wedge distal femur osteotomy (SCO) or both double osteotomy (DOT) were included. Clinical results after a varusproducing osteotomy are somewhat better described, but there is a lot of discrepancy between these studies and most studies have a low level of evidence [1, 2, 7, 10, 11, 30, 34]. Therefore, a well-performed study with a complete analysis of kinetics and kinematics of gait in combination with valid clinical scores is necessary.

The purpose of the study was to evaluate changes in gait and clinical outcomes after a varus-producing osteotomy in patients with lateral $\mathrm{OA}$ of the knee and a valgus leg alignment and compare these to a normal control group. Based on the previous study of Collins et al. [6], who found a significant increase in knee peak adduction moment, an increase in knee adduction moment during the whole stance phase was expected. We hypothesized that all the kinetics and kinematics of gait will improve towards that of a healthy control group postoperatively due to a correction of the valgus malalignment towards a varus alignment.
Such an improvement will optimally restore cartilage loading forces and knee ligament balance and reduces progression of OA or the risk of OA. An improvement in the clinical outcomes postoperative was expected.

\section{Materials and methods}

This prospective study was carried out between 2006 and 2008, after approval of the Medical Ethical Board/Committee without an assigned number, as this study was in line with our normal protocol for operating these patients. A consecutive series of 12 patients participated in this study. Patients had been indicated for a single-level or doublelevel varus osteotomy because of lateral OA of the knee and a valgus alignment. Exclusion criteria were conditions other than the OA of the knee that severely influenced gait. Ten healthy control subjects participated in the study. Written informed consent was obtained prior to participation. Patients were tested preoperatively (baseline) and 1 year postoperative, whereas control subjects were only measured once. Patient characteristics at baseline and controls characteristics are presented in Table 1.

\section{Operation techniques}

Deformity analysis according to Paley and Pfeil [28] revealed a single-level femoral valgus deformity in five patients, single-level tibial valgus deformity in three patients and double-level valgus deformity in four patients. Planning of deformity correction was aimed at correction of the lower leg to a neutral mechanical axis by angular correction of the deformed bone(s) to normal or into slight varus taking care of normal knee joint orientation (Fig. 1).

Table 1 Baseline parameters

\begin{tabular}{lcc}
\hline Parameter & Patients & Controls \\
\hline Number of subjects & 12 & 10 \\
Age [years (SD)] & $45(3.3)$ & $51(13.2)$ \\
Sex $(N)$ & & \\
Female & 8 & 6 \\
Male & 4 & 4 \\
Height [cm (SD)] & $176(13)$ & $174(12)$ \\
Weight [kg (SD)] & $81(14.0)$ & $76(8.9)$ \\
Side (Left/Right) & $5 / 7$ & \\
OA classification (SD) & & \\
Medial & $1.4(0.8)^{\mathrm{a}}$ \\
Lateral & $2.3(1.1)^{\mathrm{a}}$ & \\
\hline
\end{tabular}

$S D$ standard deviation, $N$ number, $\mathrm{cm}$ centimetre, $\mathrm{kg}$ kilogram, $O A$ osteoarthritis classification Kellgren and Lawrence

a $N=11$ 
All osteotomies were uniplanar closing wedge corrections, which were performed by one surgeon $(\mathrm{RvH})$, five medial closing wedge distal femur osteotomies (SCO), three medial closing wedge high tibial osteotomies (TKO) and a combination of both in the four double osteotomy patients (DOT). Preoperatively, a calibrated sawguide including a goniometer was used to enable accurate wedge resections according to the preoperative planning [22]. All osteotomies were fixed with angular stable $\left(\mathrm{TomoFix}^{\circledR}\right)$ plates. Postoperative rehabilitation consisted of immediate range of motion exercises, muscle training and partial weightbearing until 6 weeks postoperative. Subsequently, full weight-bearing was started depending on pain and radiographic proof of sufficient bone healing.

\section{Clinical and radiographic outcomes}

The clinical evaluation consisted of the Visual Analogue Scale for maximum pain (VAS-pm) and the frequency (VAS-pf) the patient experienced pain, The Dutch Western Ontario and McMaster Universities osteoarthritis index (WOMAC) [31], the Oxford Knee Score (OKS) [13], the Knee Outcome Survey Activities of Daily Living Scale (KOS) [16] and an evaluation of postoperative complications and reoperations. Whole leg standing anteroposterior radiographs were used to measure the pre- and postoperative hip knee ankle (HKA) angle, mechanical lateral distal femoral angle (mLDFA) and medial proximal femoral angle (MPTA), according to Paley and Pfeil [28]. Radiographic OA grading of the affected knee was performed by an independent investigator (NvE) using the Kellgren and Lawrence classification [18].

\section{Gait analysis methods}

The kinetics and kinematics of gait of each subject were measured using the Vicon motion analysis system (Vicon Motion Systems Ltd., Oxford, UK). The study of Koenraadt et al. [20] showed an accuracy of the system of at least $0.1 \mathrm{~mm}$. The system consisted of eight infrared cameras and a computer system for data acquisition, processing and analysis. Marker positions were sampled at $200 \mathrm{~Hz}$. Twenty reflective markers (14 $\mathrm{mm}$ in diameter) were placed according to the Helen Hayes lower limb model. Kinetic data were obtained simultaneously with the measurement of the kinematics using a Kistler force plate (Kistler Instruments, Switzerland) embedded in the floor and sampling at $2400 \mathrm{~Hz}$. All subjects were instructed to walk barefoot at a self-selected speed. Subjects had a fixed starting point so that their third step was placed on the surface of the force plate [5]. At least three acceptable trials were obtained for both the right and the left leg. The gait data were processed using Vicon Workstation (version 5.2) and the Optimized
Lower limb Gait Analysis (OLGA) model. A Woltering filtering routine with $\mathrm{MSE}=25$ was used to filter the data.

The gait parameters of interest were walking speed, stride length and foot progression angle. In addition, varus/ valgus (adduction/abduction) and flexion/extension angle and external moment of the knee and hip during the entire stance phase were obtained and subsequently normalized to stance time. Heel strike and toe-off were determined using the vertical ground reaction force with a threshold of $10 \mathrm{~N}$. The average of three trials per subject was used. For each OA patient, these parameters were calculated for the affected leg, whereas for the control group, the leg was randomly selected. The kinetics and kinematics of gait were analysed using custom written programs in Matlab. The accuracy of the used method in assessing the kinematics and kinetics of gait is $<5$ degrees as has been reported in the literature [24, 41].

\section{Statistical analysis}

Total test scores [mean, standard deviation (SD)] for the continuous variables (HKA angle, WOMAC, KSS, OKS, KOS, VAS-pm, VAS-pf) were calculated at baseline (preoperative) and 1 year postoperative. A paired $t$ test was used to indicate differences between the preoperative and postoperative clinical outcomes and the gait characteristics walking velocity, stride length and foot progression angle.

A Wilcoxon signed-rank test was used to test for significant differences in knee and hip angles and moments between the pre- and postoperative condition for each per cent of the stance phase. Differences between the patients and controls were tested using a Mann-Whitney $U$ test. To study the relation between the degree of deformity correction with the knee adduction moment, the mean knee adduction moment over the stance phase was first calculated. Subsequently, the Pearson correlation coefficient between the correction of HKA angle and increase of mean knee adduction moment was calculated. The effect of the three different surgical interventions on kinetics and kinematics were also analysed. However, no statistical analysis has been performed on these data because the subgroups consisted of only a few patients. A $p<0.05$ was considered significant. All data were statistically analysed with SPSS version 18.0.

\section{Results}

Not all patients had a complete data set. One patient had no preoperative clinical measurements and was therefore left out in the analysis of clinical outcomes. Two patients had an incomplete radiographic file and were therefore left out in the radiographic analysis. 


\section{Clinical and radiographic outcomes, complications and reoperations}

Postoperative all clinical results significantly improved (Table 2). The radiographic measurements showed a mean HKA angle correction of $10.4^{\circ}\left(95 \%\right.$ CI $\left.6.4^{\circ}-14.4^{\circ}\right)$. The mean mLDFA and MPTA are also shown in Table 2. In five patients (three DOT and two SCO), the hardware was removed within 1 year. One patient (SCO) underwent a pseudoarthrosis repair 6 months postoperative. No intraoperative or postoperative complications that could interfere with postoperative gait were found.

\section{Gait analysis}

\section{Spatiotemporal parameters}

The spatiotemporal parameters are shown in Table 3. Surgery did not affect the walking velocity of the patients, leaving a significant difference with the control group after surgery $(p=0.024)$. Although the stride length did not increase postoperatively, stride length after surgery was not significantly different from controls $(p=0.13)$. There was also no significant difference in foot progression angle between the preoperative, postoperative measurements and the controls.

\section{Knee and hip kinematics}

The valgus/varus and flexion/extension angle of the knee for the preoperative condition, the postoperative condition and the control group are shown in Fig. 2. Although the valgus angle of the patients significantly decreased postoperatively (except for late stance), patients had significantly more knee valgus angle during the entire stance phase before and after surgery compared to healthy controls.

Patients had pre- and postoperatively significantly less knee flexion around toe strike and less knee extension around heel off compared to controls. Knee flexion/extension angle was not significantly different between pre- and postoperative. The three types of surgery (TKO, SCO and DOT) influenced the knee angles in almost a similar manner as can be seen in the lower panels of Fig. 2.

Table 2 Clinical and radiographic outcomes

\begin{tabular}{|c|c|c|c|c|}
\hline Parameter & $\begin{array}{l}\text { Mean preoperative scores (SD) } \\
N=11\end{array}$ & $\begin{array}{l}\text { Mean postoperative scores (SD) } \\
N=12\end{array}$ & $\begin{array}{l}\text { Mean difference }(95 \% \mathrm{CI}) \\
N=11\end{array}$ & $p$ value $N=11$ \\
\hline HKA $\left(^{\circ}\right)$ & $9.3(5.7)$ valgus $^{\mathrm{b}}$ & $1.1(2.3)$ varus $^{\mathrm{a}}$ & $10.4(6.4-14.4)$ & $p=0.000$ \\
\hline $\operatorname{mLDFA}\left({ }^{\circ}\right)$ & $85.0(6.2)^{\mathrm{b}}$ & $90.0(2.0)^{\mathrm{a}}$ & $4.0(0.1-7.9)$ & n.s. \\
\hline MPTA $\left(^{\circ}\right)$ & $88.0(7.7)^{b}$ & $89.0(2.8)^{\mathrm{a}}$ & $0.0(-4.4$ to 4.4$)$ & n.s. \\
\hline WOMAC (0-96) & $57(13)$ & $81(16)$ & $-26(-33$ to -19$)$ & $p=0.000$ \\
\hline VAS-pm $(0-100)$ & $60(19)$ & $33(21)$ & $26(11-41)$ & $p=0.003$ \\
\hline VAS-pf (0-100) & $71(21)$ & $27(22)$ & $26(61)$ & $p=0.000$ \\
\hline KOS $(0-100 \%)$ & $56(15)$ & $75(15)$ & $20(35-4.2)$ & $p=0.018$ \\
\hline OKS (12-60) & $33(8)$ & $21(8)$ & $12(6.8-17)$ & $p=0.000$ \\
\hline
\end{tabular}

$S D$ standard deviation, $C I$ confidence interval, $N$ number, $O A$ osteoarthritis classification Kellgren and Lawrence, $H K A$ hip knee ankle angle, mLDFA mechanical lateral distal femoral angle, MPTA medial proximal tibial angle, WOMAC Dutch Western Ontario and McMaster Universities osteoarthritis index, VAS-pm Visual Analogue Scale for maximum pain, VAS-pf Visual Analogue Scale for how frequent the patient experienced pain, KOS Knee Outcome Survey Activities of Daily Living Scale, OKS Oxford Knee Score

${ }^{\text {a }} N=10$

b $N=12$

Table 3 Spatiotemporal parameters

\begin{tabular}{llllll}
\hline Spatiotemporal parameters & $\begin{array}{l}\text { Mean preoperative scores } \\
(\mathrm{SD}) N=12\end{array}$ & $\begin{array}{l}\text { Mean scores control group } \\
(\mathrm{SD}) N=10\end{array}$ & $p$ value $^{\mathrm{a}}$ & $\begin{array}{l}\text { Mean postoperative scores }_{(\mathrm{SD})} N=12 \\
p \text { value }^{\mathrm{b}}\end{array}$ \\
\hline Walking velocity $(\mathrm{m} / \mathrm{s})$ & $0.95(0.09)$ & $1.25(0.15)$ & $p<0.001$ & $0.93(0.25)$ & n.s. \\
Stride length $(\mathrm{m})$ & $1.10(0.18)$ & $1.38(0.14)$ & $p=0.004$ & $1.17(0.39)$ & n.s. \\
Foot progression angle $\left({ }^{\circ}\right)$ & $7.0^{\circ}\left(3.8^{\circ}\right)$ & $6.4^{\circ}\left(2.5^{\circ}\right)$ & n.s. & $5.8^{\circ}\left(3.6^{\circ}\right)$ & n.s. \\
\hline
\end{tabular}

$S D$ standard deviation, $N$ number $n . s$. nonsignificant

${ }^{a}$ Difference between preoperative scores and scores control group

${ }^{\mathrm{b}}$ Difference between preoperative and postoperative scores 

flexion/extension angles. Upper panels the knee angles of the controls, preoperative condition and postoperative condition for the valgus/varus (left panel) and flexion/extension (right panel) angle. Dark areas in the bars right above the $x$-axis indicate significant differences $(p<0.05)$ between: \&, postoperative and controls; \#, preoperative and controls; $\$$, pre- and postoperative. Lower panels knee varus/valgus and flexion/ extension angles for the DOT, SCO and TKO group. Pre- and postoperative as well as the control data are displayed. $H S$ heel strike, $T S$ toe strike, $H O$ heel off, $O H$ opposite heel strike, TO toe-off, Deg degrees, Pre preoperative, Post postoperative, $D O T$ double osteotomy, $S C O$ supracondylar osteotomy, $T K O$ high tibial osteotomy
Fig. 2 Knee valgus/varus and
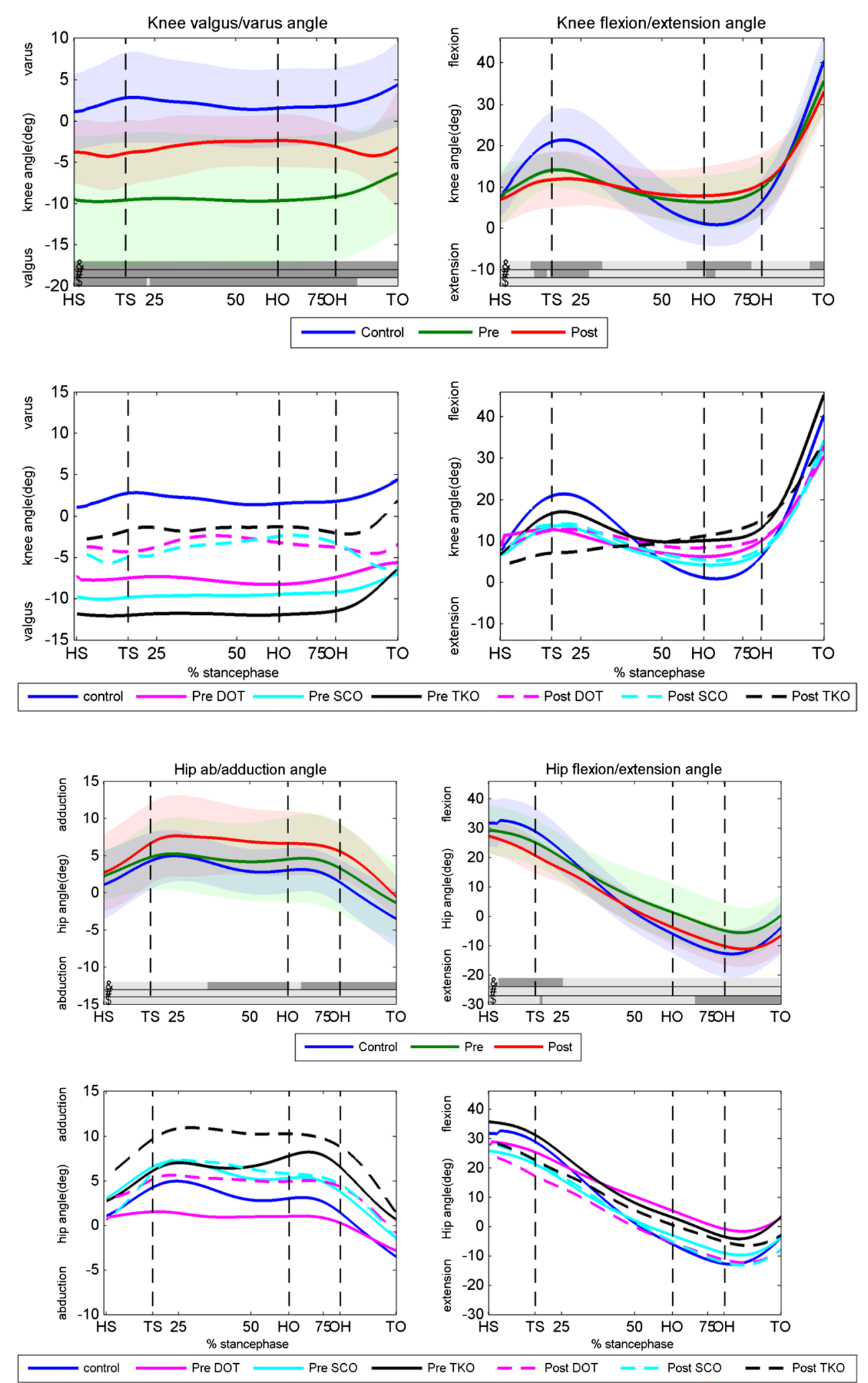

Fig. 3 Hip abduction/adduction and flexion/extension angles. Upper panels the hip angles of the controls, preoperative condition and postoperative condition for the abduction/adduction (left panel) and flexion/extension (right panel) angle. Dark areas in the bars right above the $x$-axis indicate significant differences $(p<0.05)$ between: $\&$, postoperative and controls, \#, preoperative and controls, \$, pre- and postoperative. Lower panels hip abduction/adduction and flexion/extension angles for the DOT, SCO and TKO group. Pre- and postoperative as well as the control data are displayed. $H S$ heel strike, $T S$ toe strike, $\mathrm{HO}$ heel off, $\mathrm{OH}$ opposite heel strike, $T O$ toe-off, Deg degrees, Pre preoperative, Post postoperative, DOT double osteotomy, SCO supracondylar osteotomy, TKO high tibial osteotomy
There was no significant difference in hip flexion/extension angle between the preoperative condition and the controls (Fig. 3). After surgery, the hip flexion/extension angle was significantly lower at the final part of the stance phase compared to the preoperative condition. The patients had their hip significantly more extended at the first $25 \%$ of the 
stance phase and more adducted from 35 to $100 \%$ of the stance phase in the postoperative condition compared to the controls.

\section{Knee and hip kinetics}

The external knee and hip joint moments for the pre- and postoperative condition and the controls are shown in Figs. 4 and 5, respectively. The patients had a significant lower knee adduction moment before surgery compared to healthy controls, which increased significantly postoperative during almost the entire stance phase. The mean knee adduction moment increased significantly from 0.004 preoperative to $0.204 \mathrm{Nm} / \mathrm{kg}$ postoperative $(p=0.004)$. A power calculation based on the change in mean knee adduction moment revealed a power of $95.4 \%$. After surgery, patients had only a significant lower adduction moment compared to controls around toe strike. The patients had significantly lower knee flexion moment at the first $35 \%$ of the stance phase and lower knee extension moment at toe-off (right upper panel, Fig. 4) compared to the controls. Surgery did not affect the knee flexion/extension moment.

Hip external abduction/adduction had almost no differences between the patients (pre- and postoperative) and the controls (Fig. 5). Surgery caused a significant decrease in hip extension moment around toe strike and toe-off. The hip extension moment was significantly lower after surgery at the first $50 \%$ of stance and at toe-off compared to controls. No clear differences were found between the three surgical techniques in knee and hip moments (lower panels of Figs. 4, 5).

A significant correlation was found between the correction of HKA angle and increase in mean knee adduction moment $(r=0.65 ; p=0.04)$.

\section{Discussion}

The most important finding of the present study is the significant increase in knee adduction moment during the whole stance phase postoperatively to an almost similar pattern as was found in the control group (left upper panel Fig. 4). In addition to the increase in peak adduction moment (mean change was $0.72 \% \mathrm{BW}^{*} \mathrm{Ht}(95 \% \mathrm{CI}$ $0.42,1.02)$ described by Collins et al. [6], an increased mean adduction moment during the whole stance phase of $0.20 \mathrm{Nm} / \mathrm{kg}$ after three types of osteotomies was found. Collins et al. [6] investigated the gait of a subgroup of 12 patients after a lateral opening wedge high tibial osteotomy for a mild valgus malalignment [mean HKA angle $2.4^{\circ}$
Fig. 4 Knee external knee abduction/adduction and flexion/extension moments. Upper panels the knee moments of the controls, preoperative condition and postoperative condition for the abduction/adduction (left panel) and flexion/extension (right panel) moment. Dark areas in the bars right above the $x$-axis indicate significant differences $(p<0.05)$ between: $\&$, postoperative and controls, \#, preoperative and controls, \$, pre- and postoperative. Lower panels knee abduction/adduction and flexion/extension moments for the DOT, SCO and TKO group and the controls. $H S$ heel strike, $T S$ toe strike, $\mathrm{HO}$ heel off, $\mathrm{OH}$ opposite heel strike, TO toe-off, Deg degrees, Pre preoperative, Post postoperative, DOT double osteotomy, $S C O$ supracondylar osteotomy, TKO high tibial osteotomy
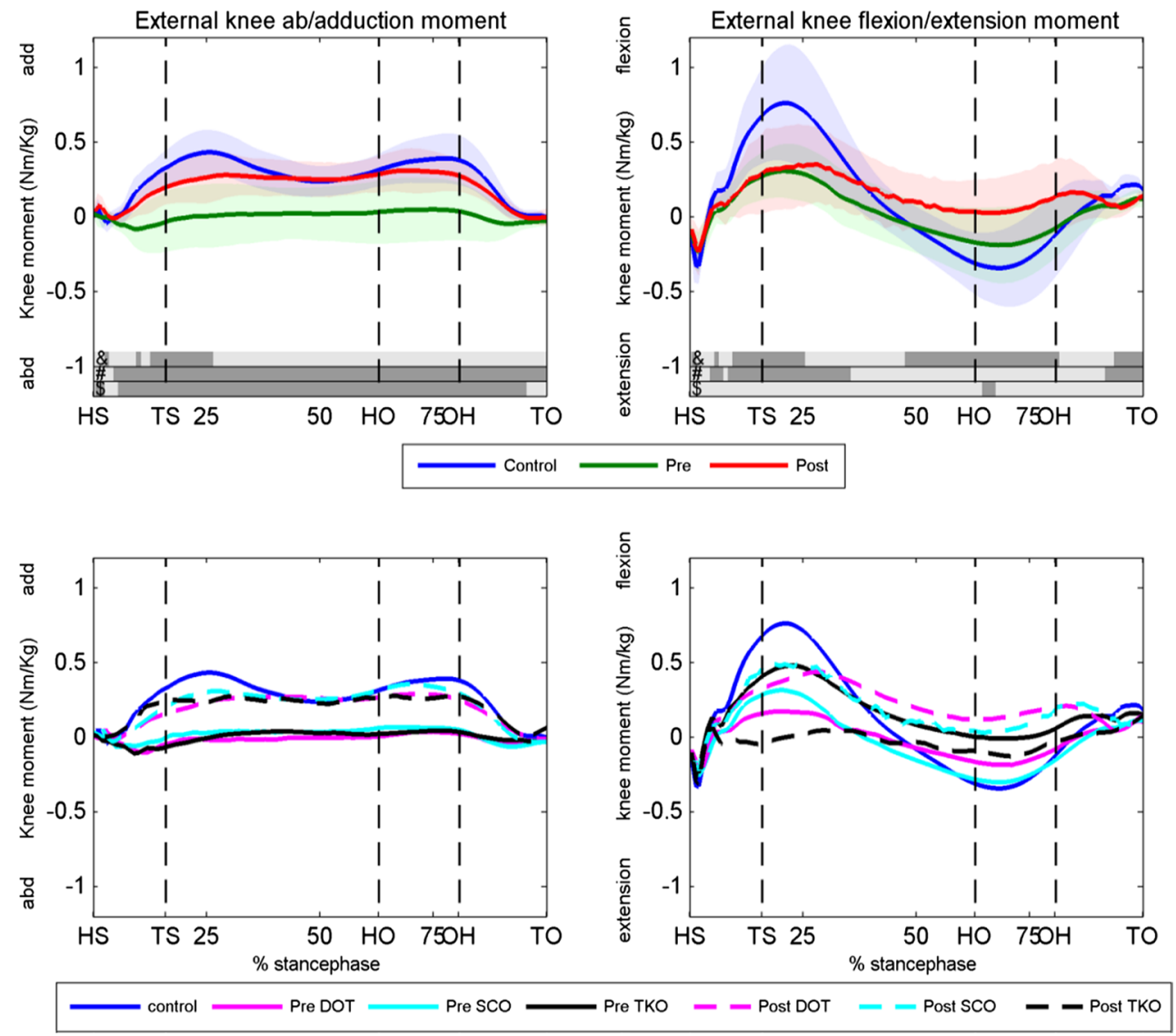
Fig. 5 Hip abduction/adduction and flexion/extension moments. Upper panels the hip moments of the controls, preoperative condition and postoperative condition for the abduction/adduction (left panel) and flexion/extension (right panel) moment. Dark areas in the bars right above the $x$-axis indicate significant differences $(p<0.05)$ between $\&$, postoperative and controls, $\#$, preoperative and controls, \$, pre,- and postoperative. Lower panels hip abduction/adduction and flexion/extension moments for the DOT, SCO and TKO group and controls. $H S$ heel strike, $T S$ toe strike, $H O$ heel off, $\mathrm{OH}$ opposite heel strike, TO toe-off, Deg degrees, Pre preoperative, Post postoperative, DOT double osteotomy, $S C O$ supracondylar osteotomy, TKO high tibial osteotomy
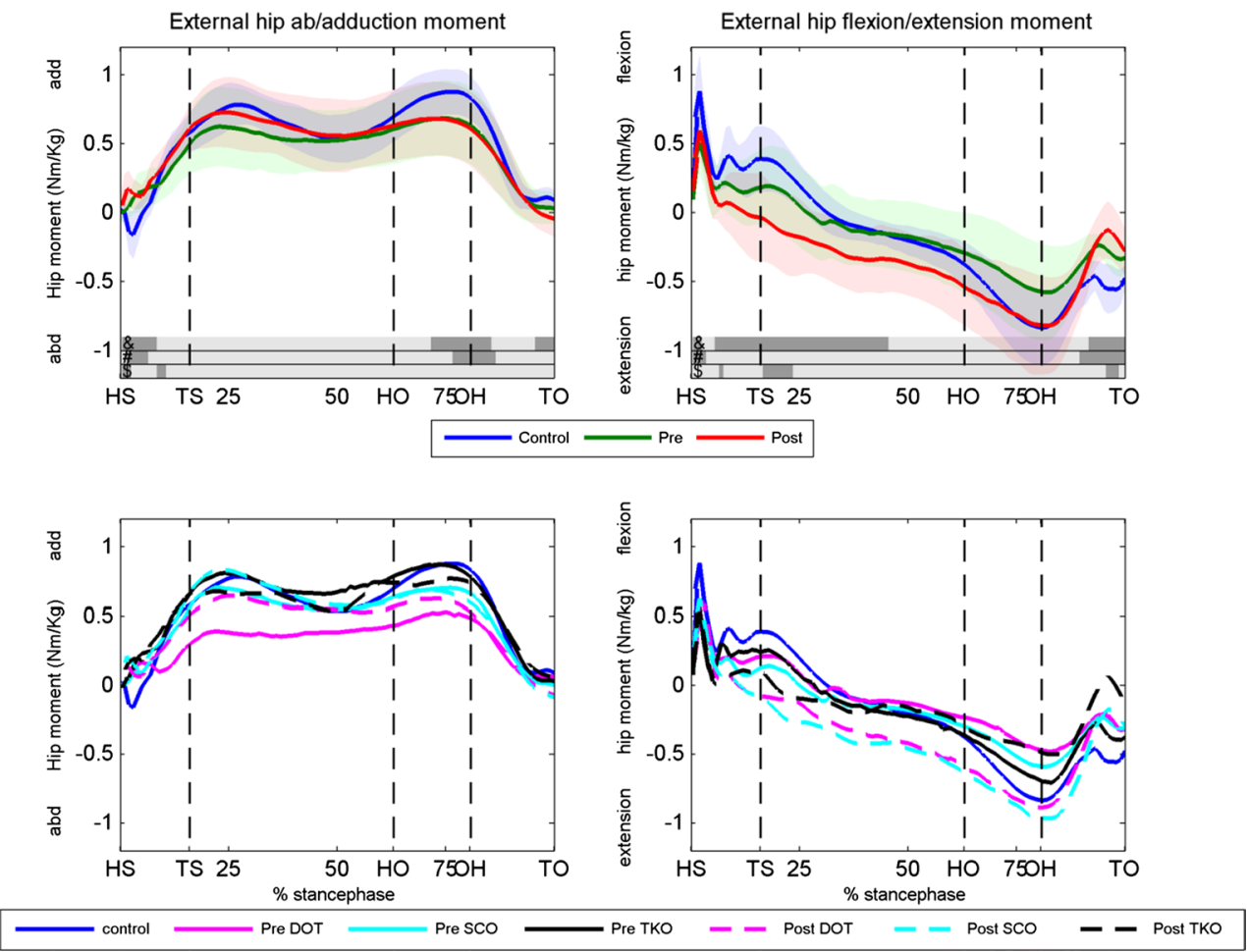

(SD 2.4)]. The authors excluded patients with an abnormal mLDFA, and they did not compare the results to a control group. In contrast to Collins et al. [6], patients with a large HKA angle [mean HKA angle 9.3 (SD 5.7)] an abnormal mLDFA were included and compared to a control group. The knee adduction moment changed postoperatively during the whole stance phase to an almost similar pattern as the control group. Our control group showed comparable results with the gait characteristics of other healthy subjects in the literature $[12,35]$.

As yet to our knowledge only one gait study has been performed with varus osteotomies [6], the mechanical axis seems the best predictor of the peak abduction/adduction moment, as shown in earlier studies with valgus osteotomies [8, 14, 38, 39]. Turcot et al. [35] found that subjects with varus knees had larger peak knee adduction moments than subjects with neutral or valgus knees. A valgus osteotomy causes an increase in the abduction moment and a lateral shift in the dynamic knee joint loading [8, 36, 38, 39]. Postoperatively, a mean correction of $10.4^{\circ}(95 \% \mathrm{CI}$ 6.4-14.4) towards a mean HKA angle of $1.1^{\circ}$ (SD 2.3) varus and an increase in the adduction moment comparable to that of healthy controls during the entire stance phase were found. It seems that a varus osteotomy, which has an opposite effect as compared to a valgus osteotomy, caused a medial shift in the dynamic knee joint load. The medial shift will optimally restore cartilage loading forces and knee ligament balance and possibly reduces the risk of OA. The mean increase in knee adduction moment during stance showed a significant correlation with the correction of the valgus malalignment.

It was showed that the other gait kinetics and kinematics improved towards that of a healthy control group after surgery, with exception of the knee and hip flexion/extension motion and moment. In general, our patients showed less knee and hip flexion/extension motion and moment during gait compared to the controls. Postoperatively these curves hardly changed compared to preoperatively. The pre- and postoperative differences between the patients and the controls could be explained by the lower walking velocities, which were significantly different between the patients and the controls. Kirtley et al. [19] already showed that the peak knee flexion moment is strongly related to walking speed. Also Brinkmann and Perry [3] found a positive correlation between knee flexion and gait velocity.

Significant improvements in WOMAC, KOS, OKS and VAS-pm and VAS-pf were found in all patients. These clinical results are comparable with the literature, although there are a lot of discrepancies between studies and most studies have a low level of evidence [1, 2, 7, 10, 11, 30, 34]. The significant improvements of all clinical outcomes prove the effectiveness of a varus-producing osteotomy; however, long-term results are needed to confirm this conclusion.

A common limitation in studying patients with knee $\mathrm{OA}$ and a valgus alignment is the low prevalence of these patients [35]. In the current study, a small number of patients (12 in total) was evaluated. Nevertheless, 
the power appeared to be 0.95 for the difference in mean abduction moment. Another limitation is that three different operation techniques were used. However, different operation techniques are needed to maintain a normal knee joint line orientation after correction of each type of bone deformity. Maintaining a normal knee joint line orientation will optimally restore cartilage loading forces and knee ligament balance $[2,28]$ after correction, and this results in long-term survival of the osteotomies [2]. Although it was not possible to perform a statistical analysis of these three subgroups, the clinical evaluations, radiographic measurements as well as the kinetics and kinematics of gait were similar in all three operation techniques; therefore, the osteotomy type chosen does not seem to influence the outcome. The operated leg was compared to a control group, instead of the healthy leg. In most studies, the operated leg is compared to the healthy leg. However, the leg deformities in our study group were large, and compensatory mechanisms during gait could have been expected in the gait cycle of the contralateral leg. Therefore, the gait patterns of the operated leg were compared with that of a healthy control group in order to be able to compare it with a healthy gait cycle.

\section{Conclusion}

This study showed that different types of varus-producing osteotomies in patients with lateral knee $\mathrm{OA}$ and a valgus alignment are a successful treatment in correcting alignment resulting in an increase in all postoperative clinical outcomes. A significant increase in the knee adduction moment was found during stance phase postoperatively, which was related to the degree of correction. Several other gait characteristics significantly changed towards that of the healthy controls. The HKA angle towards zero degrees caused a medial shift in the dynamic knee loading. The medial shift will optimally restore cartilage loading forces and knee ligament balance and reduces progression of OA or the risk of OA.

\section{Compliance with ethical standards}

Conflict of interest The authors declare that they have no conflict of interest.

Open Access This article is distributed under the terms of the Creative Commons Attribution 4.0 International License (http://creativecommons.org/licenses/by/4.0/), which permits unrestricted use, distribution, and reproduction in any medium, provided you give appropriate credit to the original author(s) and the source, provide a link to the Creative Commons license, and indicate if changes were made.

\section{References}

1. Aglietti P, Menchetti PP (2000) Distal femoral varus osteotomy in the valgus osteoarthritic knee. Am J Knee Surg 13(2):89-95

2. Babis GC, An KN, Chao EY et al (2002) Double level osteotomy of the knee: a method to retain joint-line obliquity. J Bone Joint Surg Am 84-A:1380-1388

3. Brinkmann JR, Perry J (1985) Rate and range of knee motion during ambulation in healthy and arthritic subjects. Phys Ther 65:1055-1060

4. Brouwer RW, Huizinga MR, Duivenvoorden T, van Raaij TM, Verhagen AP, Bierma-Zeinstra SMA, Verhaar JAN (2014) Osteotomy for treating knee osteoarthritis (Review). Cochrane Database Syst Rev 13(12):CD004019

5. Bus SA, de Lange A (2005) 1,2,3 step protocol: a comparison of the 1-step, 2-step, and 3-step protocols for obtaining barefoot plantar pressure data in the diabetic neuropathic foot. Clin Biomech 20(9):892-899

6. Collins B, Getgood A, Alomar AZ, Giffin JR, Willits K, Fowler PJ, Birmingham TB, Litchfield RB (2013) A case series of lateral opening wedge high tibial osteotomy for valgus malalignment. Knee Surg Sports Traumatol Arthrosc 21:152-160

7. de Carvalho LH Jr, Temponi EF, Soares LF, Gonçalves MB, Costa LP (2014) Physical activity after distal femur osteotomy for the treatment of lateral compartment knee osteoarthritis. Knee Surg Sports Traumatol Arthrosc 22:1607-1611

8. Demeo PJ, Johnson ME (2010) Midterm follow-up of openingwedge high tibial osteotomy. Am J Sports Med 38:2077-2084

9. Dewilde TR, Dauw J, Vandenneucker H, Bellemans J (2013) Opening wedge distal femoral varus osteotomy using the Puddu plate and calcium phosphate bone cement. Knee Surg Sports Traumatol Arthrosc 21(1):249-254

10. Drexler M, Gross A, Dwyer T, Safir O, Backstein D, Chaudhry H, Goulding A, Kosashvili Y (2015) Distal femoral varus osteotomy combined with tibial plateau fresh osteochondral allograft for post-traumatic osteoarthritis of the knee. Knee Surg Sports Traumatol Arthrosc 23:1317-1323

11. Forkel P, Achtnich A, Metzlaff S, Zantop T, Petersen W (2015) Midterm results following medial closed wedge distal femoral osteotomy stabilized with a locking internal fixation device. Knee Surg Sports Traumatol Arthrosc 23:2061-2067

12. Foroughi N, Smith R, Vanwanseele B (2009) The association of external knee adduction moment with biomechanical variables in osteoarthritis: a systematic review. Knee 16(5):303-309

13. Haverkamp D, Breugem SJ, Sierevelt IN, Blankevoort L, van Dijk CN (2005) Translation and validation of the Dutch version of the Oxford 12-item knee questionnaire for knee arthroplasty. Acta Orthop 76(3):347-352

14. Hurwitz DE, Ryals AB, Case JP, Block JA, Andriacchi TP (2002) The knee adduction moment during gait in subjects with knee osteoarthritis is more closely correlated with static alignment than radiographic disease severity, toe out angle and pain. $\mathrm{J}$ Orthop Res 20(1):101-107

15. Iorio R, Healy WL (2003) Unicompartmental arthritis of the knee. J Bone Joint Surg Am 85:1351-1364

16. Jrrgang JJ, Snyder-Mackler L, Wainner R, Fu DH, Harner CD (1998) Development of a patient-reported measure of function of the knee. J Bone Joint Surg Am 80(8):1132-1145

17. Kaufman KR, Hughes C (2001) Gait characteristics in patients with knee osteoarthritis. J Biomech 34:907-915

18. Kellgren JH, Lawrence JS (1956) Radiological assessment of osteo-arthrosis. Ann Rheum Dis 16(4):494-503

19. Kirtley C, Whittle MW, Jefferson RJ (1985) Influence of walking speed on gait parameters. J Biomed Eng 7(4):282-288 
20. Koenraadt KLM, Stolwijk NM, van den Wildenberg D, Duysens J, Keijsers NLW (2012) Effect of a metatarsal pad on the forefoot during gait. J Am Pediatr Med Assoc 102(1):18-24

21. Lind M, McClelland J, Wittwer JE, Whitehead TS, Feller JA, Webster KE (2013) Gait analysis of walking before and after medial opening wedge high tibial osteotomy. Knee Surg Sports Traumatol Arthrosc 21:74-81

22. Lobenhoffer P, Agneskirchner JD, Galla M (2007) Die Suprakondyläre varisierende Femurosteotomie mit speziellem Plattenfixateur. In: Lobenhoffet P (ed) Kniegelenknahe Osteotomien. Georg Thieme Verlag, Stuttgart, pp 93-106

23. Marti RK, Verhagen RA, Kerkhoffs GM, Moojen TM (2001) Proximal tibial varus osteotomy. Indications, technique, and five to twenty-one-year results. J Bone Joint Surg Am 83-A(2):164-170

24. McGinley JL, Baker R, Wolfe R, Morris ME (2009) The reliability of three-dimensional kinematic gait measurements: a systematic review. Gait Posture 29(3):360-369

25. McMahon M, Block JA (2003) The risk of contralateral total knee arthroplasty after knee replacement for osteoarthritis. J Rheumatol 30:1822-1824

26. Miyazaki T, Wada M, Kawahara H, Sato M, Baba H, Shimada $S$ (2002) Dynamic load at baseline can predict radiographic disease progression in medial compartment knee. Osteoarthritis. Ann Rheum Dis 61:617-622

27. Omidi-Kashani F, Hasankhani IG, Mazlumi M, Ebrahimzadeh MH (2009) Varus distal femoral osteotomy in young adults with valgus knee. J Orthop Surg Res 13(4):15

28. Paley D, Pfeil J (2000) Principles of deformity correction around the knee. Orthopäde 29(1):18-38

29. Prodromos CC, Andriacchi TP, Galante JO (1985) A relationship between gait and clinical changes following high tibial osteotomy. J Bone Joint Surg Am 67:1188-1194

30. Puddu G, Cipolla M, Cerullo G, Franco V, Giannì E (2007) Osteotomies: the surgical treatment of the valgus knee. Sports Med Arthrosc 15(1):15-22
31. Roorda LD (2004) Satisfactory cross cultural equivalence of the Dutch WOMAC in patients with hip osteoarthritis waiting for arthroplasty. Ann Rheum Dis 63:36-42

32. Sharma L, Hurwitz D, Thonar E, Sum J (1998) Knee adduction moment, serum hyaluronan level, and disease severity in medial tibiofemoral osteoarthritis. Arthritis Rheum 41(7):1233-1240

33. Sharma L, Song J (2001) The role of knee alignment in disease progression and functional decline in knee osteoarthritis. JAMA 286(22):188-195

34. Sherman C, Cabanela ME (2010) Closing wedge osteotomy of the tibia and the femur in the treatment of gonarthrosis. Int Orthop 34(2):173-184

35. Turcot K, Armand S, Lübbeke A, Fritschy D, Hoffmeyer P, Suvà D (2013) Does knee alignment influence gait in patients with severe knee osteoarthritis? Clin Biomech 28:34-39

36. Wada M, Imura S, Nagatani K, Baba H, Shimada S, Sasaki S (1998) Relationship between gait and clinical results after high tibial osteotomy. Clin Orthop Relat Res 354:180-188

37. Wang JW, Hsu CC (2005) Distal femoral varus osteotomy for osteoarthritis of the knee. J Bone Joint Surg Am 87(1):127-133

38. Wang JW, Ken NK (1990) The influence of walking mechanics and time on the results of proximal tibial osteotomy. J Bone Joint Surg 72(6):905-909

39. Weidenhielm L, Svensson K, Broström L-A, Rudberg U (1992) Change in adduction moment about the knee after high tibial osteotomy and prosthetic replacement in osteoarthrosis of the knee. Clin Biomech 7:91-96

40. Weidow J, Tranberg R (2006) Hip and knee joint rotations differ between patients with medial and lateral knee osteoarthritis: gait analysis of 30 patients and 15 controls. J Orthop Res 24(9):1890-1899

41. Wilken JM, Rodriguez KM, Brawner M, Darter BJ (2012) Reliability and minimal detectible change values for gait kinematics and kinetics in healthy adults. Gait Posture 35(2):301-307 